\title{
Synthesis and Self-Assembly of an Amphiphilic Poly(phenylene ethynylene) Ionomer
}

\section{Supplemental Information}

Characterization of Synthetic Intermediates

1: ${ }^{1} \mathrm{H}$ NMR (500 MHz, $d_{6}$-DMSO): $\delta(\mathrm{ppm}) 5.26(\mathrm{OH}, \mathrm{br}), 7.15(\mathrm{~s}, 2 \mathrm{H})$.

2: ${ }^{1} \mathrm{H}$ NMR $\left(500 \mathrm{MHz}, \mathrm{CDCl}_{3}\right): \delta(\mathrm{ppm}) 0.89(\mathrm{t}, J=7.0 \mathrm{~Hz}, 3 \mathrm{H}), 1.29-1.37(\mathrm{~m}, 8 \mathrm{H}), 1.46-$ $1.52(\mathrm{~m}, 2 \mathrm{H}), 1.77-1.83(\mathrm{~m}, 2 \mathrm{H}), 3.91(\mathrm{t}, J=6.4 \mathrm{~Hz}, 2 \mathrm{H}), 4.94(\mathrm{~s}, 1 \mathrm{H}), 7.02(\mathrm{~s}, 1 \mathrm{H}), 7.40(\mathrm{~s}$, 1H). ${ }^{13} \mathrm{C}$ NMR (125.7 MHz, $\left.\mathrm{CDCl}_{3}\right): \delta$ (ppm) 14.10, 22.65, 26.02, 29.10, 29.20, 29.23, 31.79, $70.39,84.35,87.59,120.94,124.80,149.82,152.66$. HRMS: calcd for $\mathrm{C}_{14} \mathrm{H}_{20} \mathrm{I}_{2} \mathrm{O}_{2}\left[\mathrm{M}^{+}\right]$ 473.9553, found 473.9567.

3: ${ }^{1} \mathrm{H}$ NMR $\left(500 \mathrm{MHz}, \mathrm{CDCl}_{3}\right): \delta(\mathrm{ppm}) 0.89(\mathrm{t}, J=6.9 \mathrm{~Hz}, 3 \mathrm{H}), 1.25-1.34(\mathrm{~m}, 8 \mathrm{H}), 1.49(\mathrm{q}, J$ $=7.3 \mathrm{~Hz}, 2 \mathrm{H}), 1.80(\mathrm{q}, J=6.6 \mathrm{~Hz}, 2 \mathrm{H}), 2.37(\mathrm{~s}, 6 \mathrm{H}), 2.78(\mathrm{t}, J=5.8 \mathrm{~Hz}, 2 \mathrm{H}), 3.92(\mathrm{t}, J=6.4 \mathrm{~Hz}$, 2H), $4.04(\mathrm{t}, J=5.8 \mathrm{~Hz}, 2 \mathrm{H}), 7.16(\mathrm{~s}, 1 \mathrm{H}), 7.21(\mathrm{~s}, 1 \mathrm{H}) .{ }^{13} \mathrm{C} \mathrm{NMR}\left(125.7 \mathrm{MHz}, \mathrm{CDCl}_{3}\right): \delta(\mathrm{ppm})$ $14.07,22.59,25.96,29.06,29.14,29.17,29.22,31.72,46.14,57.99,69.06,70.23,86.22,86.31$, 122.65, 123.15, 152.65, 153.05. HRMS: calcd for $\mathrm{C}_{18} \mathrm{H}_{30} \mathrm{I}_{2} \mathrm{NO}_{2}\left[\mathrm{MH}^{+}\right]$546.0366, found 546.0351 .

4: ${ }^{1} \mathrm{H}$ NMR (500 MHz, $\left.\mathrm{CDCl}_{3}\right): \delta(\mathrm{ppm}) 0.24(\mathrm{~s}, 9 \mathrm{H}), 0.25(\mathrm{~s}, 9 \mathrm{H}), 0.88(\mathrm{t}, J=6.8 \mathrm{~Hz}, 3 \mathrm{H})$, 1.25-1.32 (m, 8H), 1.49-1.50 (m, 2H), $1.78(\mathrm{q}, J=7.0 \mathrm{~Hz}, 2 \mathrm{H}), 2.40(\mathrm{~s}, 6 \mathrm{H}), 2.80$ (t, $J=5.5 \mathrm{~Hz}$ 2H), $3.94(\mathrm{t}, J=6.3 \mathrm{~Hz}, 2 \mathrm{H}), 4.08(\mathrm{t}, J=5.5 \mathrm{~Hz}, 2 \mathrm{H}), 6.88(\mathrm{~s}, 1 \mathrm{H}), 6.89(\mathrm{~s}, 1 \mathrm{H}) .{ }^{13} \mathrm{C} \mathrm{NMR}(125.7$ $\left.\mathrm{MHz}, \mathrm{CDCl}_{3}\right): \delta(\mathrm{ppm})-0.10,14.04,22.62,25.98,29.25,29.30,29.34,31.80,46.04,57.96$, 
$67.95,69.40,100.10,100.29,100.85,101.06,113.98,117.21,117.35,153.50,154.24$. HRMS: calcd for $\mathrm{C}_{28} \mathrm{H}_{48} \mathrm{NO}_{2} \mathrm{Si}_{2}\left[\mathrm{MH}^{+}\right]$486.3224, found 486.3224 .

5: ${ }^{1} \mathrm{H}$ NMR (500 MHz, $\left.\mathrm{CDCl}_{3}\right): \delta(\mathrm{ppm}) 0.88(\mathrm{t}, J=6.8 \mathrm{~Hz}, 3 \mathrm{H}), 1.28-1.33(\mathrm{~m}, 8 \mathrm{H}), 1.46(\mathrm{q}, J$ $=7.6 \mathrm{~Hz}, 2 \mathrm{H}), 1.79(\mathrm{q}, J=6.8 \mathrm{~Hz}, 2 \mathrm{H}), 2.36(\mathrm{~s}, 6 \mathrm{H}), 2.77(\mathrm{t}, J=5.8 \mathrm{~Hz}, 2 \mathrm{H}), 3.32(\mathrm{~s}, 1 \mathrm{H}), 3.33$ (s, 1H), $3.97(\mathrm{t}, J=6.6 \mathrm{~Hz}, 2 \mathrm{H}), 4.09(\mathrm{t}, J=5.8 \mathrm{~Hz}, 2 \mathrm{H}), 6.95(\mathrm{~s}, 1 \mathrm{H}), 6.97(\mathrm{~s}, 1 \mathrm{H}) .{ }^{13} \mathrm{C} \mathrm{NMR}$ $\left(125.7 \mathrm{MHz}, \mathrm{CDCl}_{3}\right): \delta$ (ppm) 14.09, 22.64, 25.87, 29.08, 29.20, 29.26, 31.77, 46.04, 57.98, $68.14,69.63,79.62,79.76,82.56(2), 113.28,113.41,117.70,117.98,153.67,154.25$. HRMS: calcd for $\mathrm{C}_{22} \mathrm{H}_{32} \mathrm{NO}_{2}\left[\mathrm{MH}^{+}\right]$342.2433, found 342.2430 .

6: ${ }^{1} \mathrm{H}$ NMR (500 MHz, $\mathrm{C}_{2} \mathrm{D}_{2} \mathrm{Cl}_{4}, 360 \mathrm{~K}$ ): $\delta$ (ppm) 0.92 (br s, 3H), 1.33-1.42 (br m, 10H), 1.55 (br s, 2H), 1.88 (br s, 2H), 2.47 (br s, 6H), 2.77 (br s, 2H), 4.11 (br s, 2H), 4.27 (br s, 2H), 4.23 (br s, 2H), 7.06-7.08 (br m, 2H).

8: ${ }^{1} \mathrm{H}$ NMR (500 MHz, $d_{6}$-DMSO, $360 \mathrm{~K}$ ): $\delta$ (ppm) 0.84 (br s, 3H), 1.25 (br s, 6H), 1.34 (br m, 2H), 1.53 (br m, 2H), 1.76 (br m, 2H), 3.36-3.43 (br m, 9H), 4.02-4.10 (br m, 4H), 4.62 (br m, 1H), 4.71 (br m, 1H), 7.09-7.15 (br m, 1H), 7.34-7.49 (br m, 1H). 